\title{
Understanding the biology of antigen cross-presentation for the design of vaccines against cancer
}

\section{Cynthia M. Fehres, Wendy W. J. Unger, Juan J. Garcia-Vallejo and Yvette van Kooyk*}

Department of Molecular Cell Biology and Immunology, VU University Medical Center, Amsterdam, Netherlands

Edited by:

Marianne Boes, University Medical

Centre Utrecht, Netherlands

\section{Reviewed by:}

Sven Burgdorf, Rheinische

Friedrich-Wilhelms-University,

Germany

Richard A. Kroczek, Robert

Koch-Institute, Germany

${ }^{*}$ Correspondence:

Yvette van Kooyk, Department of

Molecular Cell Biology and

Immunology, VU University Medical

Center, P.O. Box 7057, 1007 MB

Amsterdam, Netherlands

e-mail:y.vankooyk@vumc.nl
Antigen cross-presentation, the process in which exogenous antigens are presented on $\mathrm{MHC}$ class I molecules, is crucial for the generation of effector $\mathrm{CD}^{+} \mathrm{T}$ cell responses. Although multiple cell types are being described to be able to cross-present antigens, in vivo this task is mainly carried out by certain subsets of dendritic cells (DCs). Aspects such as the internalization route, the pathway of endocytic trafficking, and the simultaneous activation through pattern-recognition receptors have a determining influence in how antigens are handled for cross-presentation by DCs. In this review, we will summarize new insights in factors that affect antigen cross-presentation of human DC subsets, and we will discuss the possibilities to exploit antigen cross-presentation for immunotherapy against cancer.

Keywords: cross-presentation, dendritic cells, antigen processing and presentation, anti-cancer vaccine, $C D 8^{+} \mathrm{T}$ cells

\section{INTRODUCTION}

For the induction of antigen-specific $\mathrm{CD} 8^{+} \mathrm{T}$ cells, antigen needs to be presented in MHC class I molecules in order to be recognized by the TCR/CD3 complex on $\mathrm{CD}^{+} \mathrm{T}$ cells. Peptides derived from endogenous proteins degraded in the cytosol, that are transported into the endoplasmic reticulum (ER), are loaded on MHC class I molecules, which will be transported to the plasma membrane as a stable peptide-MHC class I complex (1). The presentation of endogenous-derived peptides allows the immune system to detect cells that present altered self peptides or foreign peptides and is therefore an important defense mechanism against cancer or viruses (2). Although peptide-MHC class I complexes can be directly recognized by naïve $\mathrm{CD} 8^{+} \mathrm{T}$ cells, these cells require adequate co-stimulation from antigen-presenting cells (APCs) in order to become potent effector $\mathrm{CD}^{+}{ }^{-} \mathrm{T}$ cells with cytotoxic potential. Besides, APCs can also encounter exogenous antigens, namely of microbial or tumor origin, which they internalize for processing and presentation in MHC class I molecules, a phenomenon known as antigen cross-presentation.

Although multiple APCs are able to cross-present antigens, dendritic cells (DCs) are the most efficient cells in vivo (3-5). The potential of DCs to cross-present antigen has initiated many research questions aimed at finding strategies to enhance crosspresentation of DCs in order to improve tumor- and viral-specific $\mathrm{CD}^{+} \mathrm{T}$ cell responses for the treatment of cancer or infectious diseases. Several questions remain unanswered, such as the molecular basis for the differences in cross-presentation efficiency observed amongst different DC subsets, in steady-state or under inflammatory conditions. In addition, recent studies also suggest that the capacity to cross-present can be influenced by the type of antigen and the presence and timing of inflammatory signals (6). This would imply that antigen cross-presentation is not a functional specialization of certain DC subsets, but a process that can occur in many APCs under specific conditions. In this review, we will discuss the factors that have been described to influence crosspresentation of various human DC subsets, and their implication in the design of immunotherapies against cancer.

\section{CELL BIOLOGY OF ANTIGEN CROSS-PRESENTATION}

A defining aspect of the adaptive immune system is its capacity to elicit antigen-specific cellular immune responses by the instruction of antigen-specific $\mathrm{CD}^{+}$and $\mathrm{CD} 8^{+} \mathrm{T}$ cells. This property is entirely based on the presentation of antigen in MHC molecules (the peptide-MHC complex) and its recognition by the $\mathrm{T}$ cell receptor. The loading of extracellular antigen in MHC-II, recognized by $\mathrm{CD} 4^{+} \mathrm{T}$ cells, occurs in a different intracellular compartment than the loading of antigen in MHC-I, recognized by $\mathrm{CD}^{+}{ }^{+} \mathrm{T}$ cells. In the case of MHC-II, after its synthesis in the ER, complexes are formed with CD74 (also known as the invariant chain) to allow proper folding, trafficking, and protection of the peptide-binding groove. CD74 helps guiding the CD74-MHC-II complex move on to the endolysosomal pathway, where late endosomal proteases such as cathepsin S and L degrade CD74 and leave MHC-II complexed to the peptide-binding groove part of CD74 (the CLIP peptide), which is later exchanged for an antigenic fragment with the help of the chaperone HLA-DM (7). Although the process leading to antigen presentation on MHC-I also involves six basic steps (8); namely, acquisition of antigens (1); tagging of the antigenic peptide for destruction (2), proteolysis (3), transport of peptides to the ER (4), loading of peptides to MHC-I molecules (5), and the display of peptide-MHC-I complexes on the cell surface (6); the variety of intracellular compartments and pathways involved in MHC-I antigen presentation is considerably more complex than that of MHC-II.

The acquisition of antigenic peptides for MHC-I presentation is a highly heterogeneous process and multiple pathways have been 


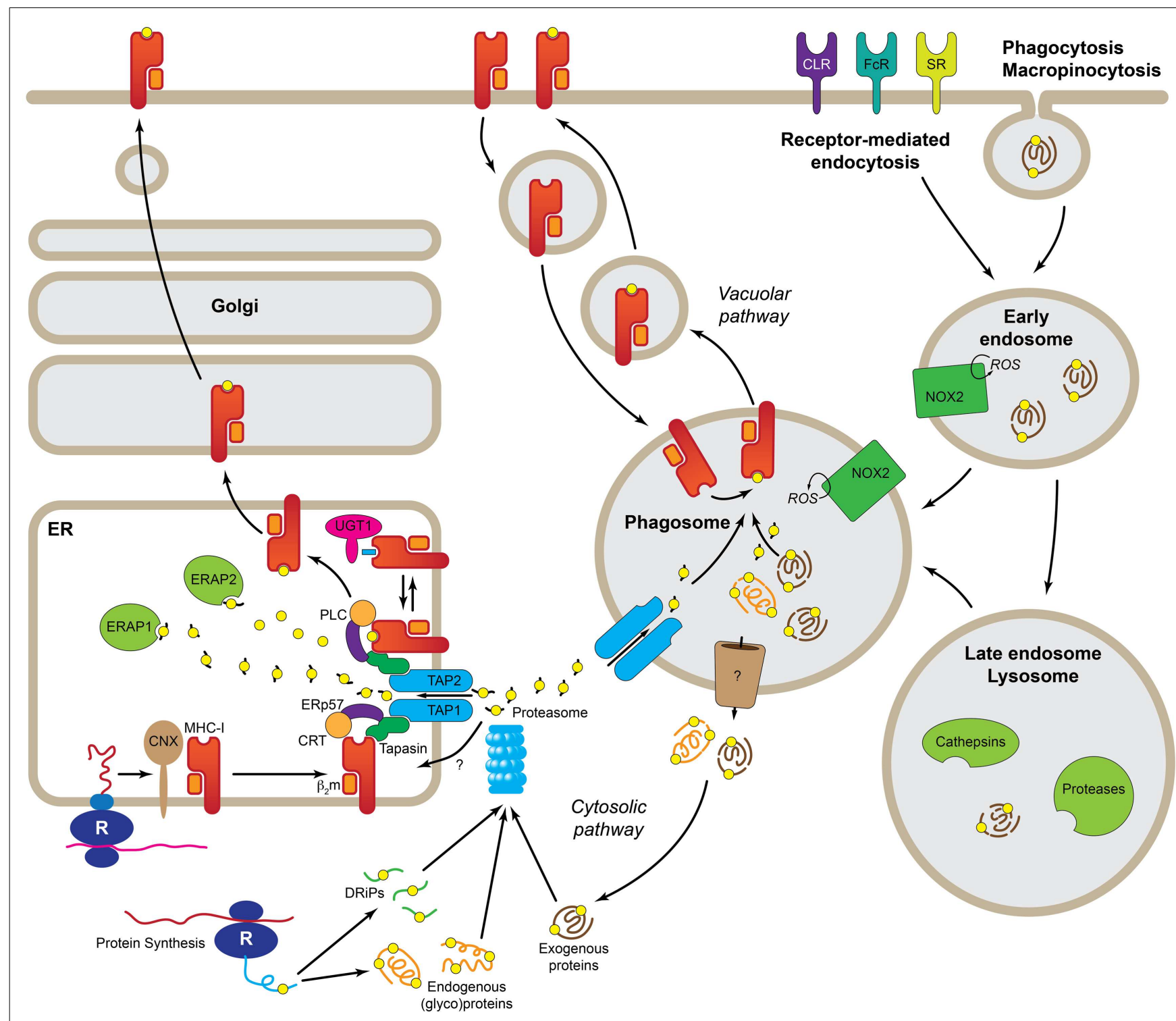

FIGURE 1 | Molecular pathways leading to cross-presentation in DCs. DCs take up Ag by three general mechanisms, receptor-mediated endocytosis, phagocytosis, or macropinocytosis. Once the Ag reaches the endolysosomal pathway, depending of the specific routing, it may be degraded by the concourse of the mild $\mathrm{pH}$ and different types of cathepsins and other proteases. At this point, properly degraded Ag can be directly loaded into recycling MHC-I in the phagosome (Vacuolar pathway). Ag that still needs further processing must be transported to the cytosol (Cytosolic pathway) where it is degraded, together with endogenous proteins and DRiPs, by the proteasome. The peptides generated by the proteasome are transported by TAP or a yet uncharacterized transporter into the ER where they are loaded into MHC-I with the help of the peptide-loading complex. Further trimming in the ER prior to loading, it is possible by the presence of ER-localized endopeptidases (ERAP1 and 2). R, ribosome; CNX, calnexin; CRT, calreticulin; b2m, b2microglobulin; UGT1, UDP-glucose:glycoprotein glucosyltransferase 1; ERAP1/2, ER-aminopeptidases 1/2; PLC, peptide-loading complex; ERp57, protein disulfide isomerase 3; TAP1/2, transporter associated with antigen-presenting 1/2; DRiPs, defective ribosomal products; ROS, reactive oxygen species; NOX2, NADPH oxidase 2; CLR, C-type lectins; FcR, Fc receptors; SR, scavenger receptors. described so far. There are two main sources of antigens for MHCI presentation, intracellular and extracellular (Figure 1). Antigenic peptides derived from cytosolic proteins, e.g., viral proteins, are the prime source of peptides for MHC-I (9), but other proteins carrying signal sequences targeting to the secretory pathway can also be presented on MHC-I, either from defective ribosomal products (or DriPs) (10) or from mature proteins (11). These mechanisms are at play on all cells expressing MHC-I. However, what makes
DCs and, to a lesser extent also macrophages and B cells, best at cross-presentation is their capacity to use extracellular antigens as source of peptides for MHC-I presentation. The uptake of extracellular antigens by APCs is achieved by three main transport pathways, namely receptor-mediated endocytosis, phagocytosis, and macropinocytosis; although there are differences in the efficiency of each of these pathways amongst DCs, B cells, and macrophages. Thus, macrophages seem to be best at phagocytosis, whereas DCs 
prefer receptor-mediated endocytosis. Amongst the many classes of receptors that mediate endocytosis of antigens are the $\mathrm{B}$ cell receptor (specific for B cells), Fc receptors, heat-shock protein receptors, scavenger receptors, and the C-type lectin receptors (CLRs). In general, these receptors mediate internalization of antigens to endosomes, however, the nature of the endosomes and their fate seems to vary for the different receptor types involved and, consequently, also their efficiency in inducing cross-presentation. Furthermore, many of the receptors involved in antigen uptake for cross-presentation are also able to mediate signaling and, in several cases, it has been demonstrated that signaling is necessary for cross-presentation. This was elegantly demonstrated in experiments where bacteria were opsonized with either antibodies or complement. Although both opsonization modalities lead to efficient phagocytosis, only the Fc receptor-mediated resulted in effective $\mathrm{CD}^{+} \mathrm{T}$ cell responses (12). Signaling through other receptors, such as the C-type lectins, Dectin-1 (13) or DNGR-1 (also known as Clec9A) (14) also enhances cross-presentation.

Both endogenous and to a minor extent exogenous antigen can thus be loaded on MHC class I. A factor that conditions the access of peptides to MHC-I is the biosynthetic pathway of the MHC-I molecule. The MHC-I complex consists of a heavy chain, a transmembrane glycoprotein with a short cytoplasmic domain that, upon translation in the ER, assembles with $\beta_{2}$-microglobulin into a heterodimer. This process is integrated with the incorporation of the peptide into the peptide-binding groove of the heavy chain, and requires the participation of the peptide-loading complex, which consists of multiple components, including the $\mathrm{ABC}$ peptide transporter TAP that allows the transport of peptides from the cytosol into the ER (15). The key concept is that to this process, the MHC-I heterodimer is stabilized until a highaffinity peptide is incorporated into the peptide-binding groove. In most cases, cross-presentation is TAP- and proteasome-dependent (16), also called the cytosolic pathway. The proteasome is a selfcompartmentalized, energy-dependent nanomachine that works as a protease to degrade misfolded, damaged, and inaccurately synthesized proteins (17). In the context of IFN- $\gamma$ or DC maturation (18), the proteasome undergoes structural changes in its substrate-binding pockets that contribute to optimizing the quality and quantity of the generated peptides (19). Still, peptides generated by the proteasome may require further trimming by two ER-resident aminopeptidases (20). To make it more complex, proteasome-dependent, yet TAP-independent cross-presentation has been recently described, suggesting the existence of a still unidentified peptide transporter (Figure 1) (21).

A cross-presentation pathway referred to as vacuolar uses endolysosomal proteases to degrade internalized bacteria and other antigens, frequently particulated, in order to allow loading on MHC-I molecules recycled from the extracellular membrane (22). Also proteasome-derived peptides may enter the vacuolar pathway $(23,24)$. Data obtained from $\mathrm{TAP}^{-/-} \mathrm{DCs}$, that are unable to incorporate peptides via TAP into the ER, indicates that cross-presentation is still possible, though to a lesser extent (25).

Several questions remain unsolved, such as the mechanism by which antigens are exported from endosomes into the cytosol for proteasomal degradation (9), whether hybrid organelles resulting from the recruitment of TAP and the peptide-loading complex to phagosomes and endosomes exist (26-28), and if interconnected ER-phagosomes are involved in cross-presentation (29). Regardless of this issue, evidence indicates that the accumulation of antigen in endosomes with low (but steady) proteolytic and relatively high $\mathrm{pH}$ conditions favors cross-presentation (30-32). In this respect, it has been proposed that limited antigen degradation correlates with efficient cross-presentation (30). Primarily decreased proteolysis is found in the endocytic compartments of DCs compared to other phagocytes, which is due to low levels of lysosomal proteases, or decreased protease activity. These can be regulated by high $\mathrm{pH}$ present, or high activity levels of the NADPH oxidase 2 (NOX2) in endosomal and phagosomal compartments of DCs.

HUMAN DC SUBSETS AND ANTIGEN CROSS-PRESENTATION Two main subsets of human DCs have been described: plasmacytoid DCs (pDCs) and myeloid DCs (mDCs, also known as conventional DCs). The majority of pDCs are located in the blood and their main function is the production of type I IFN upon microbial infection (33). Recent data also show that human pDCs are capable to cross-present antigens either derived from apoptotic cells (34) or when antigens are encapsulated in nanoparticles and targeted to specific uptake receptors expressed by pDCs (35). Next to pDCs, two major populations of mDCs can be identified in blood: $\mathrm{BDCA}^{+} / \mathrm{CD} 1 \mathrm{c}^{+} \mathrm{DCs}$ and $\mathrm{BDCA} 3^{+} / \mathrm{CD} 141^{+} \mathrm{DCs}$. The $\mathrm{BDCA}^{+}$DCs are described as potent inducers of $\mathrm{CD}^{+} \mathrm{T}$ cell responses in vitro and in vivo (36-39); however, it is not yet clear how this capacity relates to the other human DC subsets (39-41). A recent publication showed that blood $\mathrm{BDCA}^{+} \mathrm{DCs}$ are more potent in cross-presentation compared to $\mathrm{BDCA1}^{+}$DCs when antigens of necrotic cells or soluble antigen were given that ended up in late endosomes and lysosomes $(41,42)$. In contrast, when antigens were targeted to early endosomes, using antigens conjugated to an anti-CD40 monoclonal antibody, BDCA1 ${ }^{+}$DCs were as efficient at cross-presentation as $\mathrm{BDCA}^{+}$blood DCs. These results suggest that the capacity of DC subsets to cross-present is not intrinsic, but might also be determined by the route of antigen uptake and subsequent accumulation of the antigen in early endocytic compartments.

Due to the small number of mDCs in tissues, studies on human $\mathrm{mDC}$ have been hampered, with the exception of the human skin. Based on the expression of CD1a and CD14, the human skin contains at least three main subsets of DCs: $\mathrm{CD}^{+}{ }^{+} / \mathrm{CD} \mathrm{c}^{+}$dermal DCs (dDCS), CD14 ${ }^{+}$dDCs, and CD1a ${ }^{\text {High }}$ epidermal Langerhans cells (LCs), which all migrate to the skin-draining lymph nodes upon activation (43). LCs and CD1a ${ }^{+}$dDCs seem to be more efficient at cross-presentation, as compared to the CD14 ${ }^{+}$ dDCs $(44,45)$. In addition to the three main populations of skin DCs, a minor BDCA $3^{\text {High }} \mathrm{CD} 14^{-} \mathrm{CD} 111 c^{\text {low-int }}$ subset of DCs is recently identified in human skin, lung, and liver. Parallel phenotypic analyses suggest that these cells are potentially related to blood $\mathrm{BDCA}^{+}$DCs. The skin BDCA3 ${ }^{\text {High }}$ DCs have shown to be superior in cross-presentation of soluble antigens when compared to the other skin DC subsets, as well as compared to $\mathrm{BDCA}^{+}{ }^{+} \mathrm{DCs}, \mathrm{BDCA}^{+} / \mathrm{CD}_{1}{ }^{+} \mathrm{DCs}$, and $\mathrm{CD} 14^{+}$monocytes derived from blood (41). Care should be taken not to confuse the BDCA3 ${ }^{\text {High }}$ skin DCs described by Haniffa et al. with the 
dermal BDCA $3^{+} \mathrm{CD} 14^{+}$DCs described by Chu et al. (46). The latter are immunoregulatory tissue-resident DCs characterized by the constitutive secretion of IL-10 (46).

Altogether, findings on cross-presentation capacity of human DC subsets show that most subsets are capable to cross-present antigens. However, it becomes clear that other factors also influence the capacity to cross-present, like the antigen formulation, the mode of delivery, and the intracellular routing of the antigen, as well as the activation signals for the DCs.

\section{FACTORS DETERMINING CROSS-PRESENTATION}

The capacity of DC to cross-present antigens is not only dictated by characteristics of a given DC subset, but it starts to become clear that additional factors influence the cross-presentation capacity of these DC subsets as well. It must be mentioned however that most knowledge about human DC function is obtained from in vitro studies and thus may not fully reflect their behavior in vivo.

\section{MODE OF ANTIGEN INTERNALIZATION}

Antigens can be taken up by DCs via multiple mechanisms, including non-specific, receptor-independent processes, like pinocytosis and phagocytosis, or via specific, receptor-mediated processes such as uptake through CLRs, Fc receptors, and scavenger receptors. Blood $\mathrm{BDCA}^{+}$DCs are reported to be able to cross-present untargeted pp65 recombinant protein to a lesser extent than blood BDCA1/CD1c ${ }^{+}$DCs in vitro. However, when the cells were stimulated with polyI:C, the BDCA3 ${ }^{+}$blood DCs became more potent to cross-present the pp65 protein compared to $\mathrm{CD} 1 \mathrm{c}^{+}$blood DCs (39). These results were confirmed by Mittag et al., who showed that $\mathrm{CD}_{1 c^{+}}$blood DCs are more potent in cross-presenting soluble influenza protein without TLR stimulation, but in the presence of polyI:C the BDCA3 ${ }^{+}$blood DCs became more potent (47). Surprisingly, they also show that pDCs were able to cross-present soluble protein in the absence of polyI:C. Whether human bloodderived pDCs are capable to cross-present soluble proteins is questionable, since others provided evidence that pDCs were unable to cross-present soluble proteins in the presence and absence of TLR stimulation (48-50).

In addition, cross-presentation of NY-ESO-1 antigen administrated as antigen-antibody immune complexes (IC), allowing $\mathrm{Fc} \gamma$ receptor-mediated uptake, did not enhance antigen-specific $\mathrm{CD}^{+} \mathrm{T}$ cell responses by pDCs (50). In comparison, BDCA1 ${ }^{+}$ blood DCs cross-presented the Fc $\gamma$ receptor-targeting NY-ESO$1 /$ IC more efficiently compared to the soluble protein formulation. Another study also showed that $\mathrm{Fc} \gamma$ receptor-mediated uptake of pp65-IC enhanced the cross-presentation capacity of both the $\mathrm{BDCA}^{+}$and $\mathrm{BDCA}^{+}{ }^{+} \mathrm{DCs}$ compared to the uptake of HCMV pp65 protein $(50,51)$. These studies indicate that the mode of antigen internalization and antigen formulation have a profound impact on cross-presentation capacity.

Besides the uptake of antigen via $F c \gamma$ receptors, receptormediated uptake is also often studied using CLRs to stimulate antigen cross-presentation and $\mathrm{CD}^{+} \mathrm{T}$ cell responses. CLRs are a family of pattern-recognition receptors expressed by DCs and recognize various carbohydrate structures. Upon recognition and binding to the receptor, most CLRs respond by internalization and processing of the antigen (52). Their specific expression on certain
DC subsets and the capacity to internalize antigens, make CLRs interesting targets to induce cross-presentation.

Targeting of antigen to the CLR DCIR, which is expressed by all human DC subsets tested, including LCs and blood mDCs and pDCs, resulted in improved cross-presentation by all subsets (53). Again, the blood $\mathrm{mDC}$ subset induced the highest percentages of tetramer-positive $\mathrm{CD}^{+} \mathrm{T}$ cells, indicative of a superior capacity to cross-present antigens, also when they are taken up in a receptor-mediated fashion. However, not all receptors show the same effects on antigen cross-presentation, as shown by Cohn et al. (42). Their study showed that BDCA $3^{+}$DCs were superior in cross-presentation of antigens taken up via the CLR DEC-205, which routes antigen to late endosomes and lysosomes, compared to $\mathrm{BDCA}^{+}$DCs and pDCs. However, when antigens were delivered to early endosomes through conjugation to CD40 or $\mathrm{CD} 11 \mathrm{c}, \mathrm{BDCA}^{+}{ }^{+} \mathrm{DCs}$ and $\mathrm{pDC}$ were as efficient in antigen crosspresentation as the BDCA3 ${ }^{+}$DCs (42). Furthermore, Chatterjee et al. have shown that targeting antigen to CD40 resulted in the most efficient cross-presentation in human moDCs and BDCA1 ${ }^{+}$ DCs, despite the fact that CD40 was least efficient in antigen internalization compared to DEC-205 or mannose receptor (MR) (54). These results indicated that routing of antigen to more degradative, late endosomes, via DEC-205- or MR-mediated uptake, may have a negative effect on cross-presentation compared to antigen routing to early endosomes. Altogether, the results demonstrate that the intracellular routing of antigens is of importance for antigen cross-presentation. Thus, all human DC subsets seem to have the capacity to cross-present antigens, provided that the antigen is given in a suitable formulation under appropriate conditions.

\section{ANTIGEN FORMULATION}

The antigen form and mode of delivery is crucial in determining the efficiency of cross-presentation. As DCs encounter antigens in many sizes and shapes, derived form various sources, multiple antigens might be differently handled by DCs, which might result in modification of the intracellular routing of antigen, thereby affecting the potency to cross-present. As described above, antigen can be soluble, as synthetic long peptides, protein, or it can be included in a pathogen/viral structure, as necrotic cells or as immune complex. Alternatively, antigens can be conjugated to antibodies specific for DC uptake receptors, or glycans that interact with CLRs. These different antigen formulations may affect the size of the antigen and receptor-targeting specificity, possibly affecting the type of DC that interacts with the antigen and the mode of uptake and intracellular routing.

To achieve and promote cross-presentation, different antigen formulations have been studied, such as nanoparticles, apoptotic cells or monoclonal antibodies, or glycans conjugated to antigens as discussed earlier. Targeting antigen to DNGR-1/CLEC9a, which expression in humans and mouse is restricted to $\mathrm{CD} 8 \alpha^{+}$. like DCs (55), using PLGA nanoparticles conjugated to CLEC9a Moabs increased cross-presentation compared to isotype-control PLGA nanoparticles, implying that antigen uptake via CLEC9a enhances routing of the antigen to the cross-presentation machinery (53). Our own results show that targeting antigen to the CLR DC-SIGN using glycan- or antibody-modified liposomes results in enhanced cross-presentation capacity of DCs in vitro and in vivo 
(56). Furthermore, dendrimer technology has shown that a multivalent presentation of antigen, as well as particle size, enhances cross-presentation by DCs. Glycosylation of dendrimers enhances the DC-SIGN-mediated uptake of the particles, favoring enhanced $\mathrm{CD}^{+}$and $\mathrm{CD}^{+}{ }^{+} \mathrm{T}$ cell responses (57).

There is evidence that also for LCs, the antigen formulation is crucial in order to allow cross-presentation by LCs. It has been shown that isolated human LCs were incapable to cross-present heat-inactivated measles virus, which is specifically taken up via Langerin (58). In contrast, others have shown that skin-derived human LCs were capable to cross-prime influenza-specific CD8 ${ }^{+}$ T cells after targeting with an influenza protein conjugated to antiLangerin antibodies (48), demonstrating that there is an inconsistency whether human LCs can cross-present or not and under which circumstances. Altogether, these findings demonstrate that the formulation of antigen (either small peptides or bigger particles, like viral- or bacterial-antigens, necrotic cells, and nanoparticles) has proven to have an influence on the cross-presentation capacity of various DC subsets.

\section{ADJUVANTS AND DC MATURATION STATUS}

In general, DC maturation enhances the potency of DCs to crosspresent antigen. A large set of TLR ligands are known that act as adjuvants and stimulate cross-presentation. Because each DC subset may express a specific set of TLR receptors, they may differently respond to TLR ligands, influencing the induction of crosspresentation. For example, isolated human LCs show increased cross-presentation of antigenic peptides in the presence of the TLR3 ligand polyI:C, whereas addition of the TLR4 adjuvant LPS or the TLR7/8 adjuvant R848 does not enhance the capacity to cross-present (Fehres et al., submitted). For instance the human skin, an attractive site for vaccination because it harbors many, easy-accessible DCs, is currently studied to identify suitable adjuvants to trigger and activate skin DCs for cross-priming. We and others have shown that intradermal administration of soluble TLR ligands does not induce DC maturation as observed with in vitro generated monocyte-derived DCs (59). The discrepancy between DC maturation after TLR activation in vitro and in situ might be caused by specific, local suppression within the skin microenvironment. Ideally, the adjuvant simultaneously stimulates several cell types, resulting in a mix of activated immune cells, cytokines, and chemokines at the vaccination site. Most promising into this respect seems Aldara, an FDA-approved immune response modifier skin cream, containing 5\% of the TLR7 agonist imiquimod. Aldara is mostly used to treat non-melanoma skin tumors. Recently it was shown that application of Aldara cream results in inflammasome activation and IL-1 release by keratinocytes in naïve murine skin (60). This effect was mediated independent of TLR7 activation and attributed to isostearic acid, the major component of the vehicle. However, for induction of full inflammation, both imiquimod and the vehicle cream were shown to be required. Following topical application of Aldara skin cream to human skin explant, we observed enhanced migration and maturation of dermal DCs (Fehres et al., submitted). Combining the Aldara skin cream with Mart-1-peptide vaccination in human skin affected the migratory potential of $\mathrm{CD}_{14}{ }^{+}$skin DC, which was associated with up-regulation of co-stimulatory molecules and increased activation and IFN- $\gamma$ secretion of Mart1 -specific CD8 ${ }^{+}$T cells. Notably, the enhanced effects on DC and T cell activity were not observed when injecting soluble TLR7 and/or 8 ligands intradermally.

Besides being used as adjuvant in cancer vaccines, the aforementioned DC stimuli have also been used as stand-alone immunotherapeutics. It is anticipated that application of adjuvants at the tumor site reverses the immune-inhibitory phenotype of tumor-infiltrated DCs that ingest tumor antigens (TA), herewith restoring TA-specific $\mathrm{T}$ cell priming and anti-tumor immunity. An advantage of local delivery is a strong reduction in immune-related adverse events such as cytokine release syndrome and liver toxicity observed with systemic treatment. Indeed, topical application of the imiquimod containing cream led to residual tumors in $8 \%$ of patients in basal and squamous cell carcinoma patients (61). Furthermore, near tumor injection of low doses of agonistic anti-CD40 antibodies in a slow-release formulation was shown to activate TA-specific $\mathrm{CD}^{+} \mathrm{T}$ cells, which were able to act systemically and eradicate distant tumors (62). In addition, intra-tumoral injection of a TLR2/6 agonist spectacularly prolonged survival of pancreatic cancer patients with 9 months (63). The beneficial effects of TLR2/6 treatment were attributed to emergence of a strong immune response. Increased NK cytotoxic activity as well as elevated levels of TNF and IL- 6 were noted.

Although soluble TLR ligands do not evoke strong maturation of skin DCs when injected into the skin as adjuvant, the discovery that tumor cells express TLRs has evoked interest in application of TLR agonists as monotherapy at the tumor site (64). Administration of a TLR3 agonist in melanoma lesions limits cell proliferation directly. Additionally, combined with a protein synthesis inhibitor even tumor cell death was induced (65).

The use of intradermal injected cytokines as immunostimulators has been explored $(66,67)$. In particular, granulocytemacrophage colony-stimulating factor (GM-CSF) enhanced recruitment of DCs to the vaccine administration site, which ensures presentation of the administered TA by professional DCs and consequently priming of TA-specific T cells $(66,68)$. Furthermore, clinical trials have been conducted and/or are ongoing in which patients receive irradiated tumor cells genetically engineered to over-express GM-CSF (69). A small number of responses were demonstrated in Phase I trials in renal cell carcinoma and melanoma patients (68). However, in subsequent studies, GVAX monotherapy did not result in clinical responses. Indeed, the efficacy of GVAX might be improved by combining with immune check-point inhibitors, which aim to prevent inhibition of effector $\mathrm{T}$ cells and/or to silence Tregs. In murine pre-clinical models, GVAX combined with anti-CTLA-4 treatment enhanced efficacy and tumor regression in the B16 melanoma model, along with the presence of certain toxicities, such as skin depigmentation (70). Recently, a phase I study was completed showing dose escalation and safety, warranting further investigation of treating patients with this combination. Alternatively, GVAX has been combined with chemotherapeutic agents such as cyclophosphamide, which is currently being tested in clinical trials in metastatic melanoma patients. However, chemotherapy has been associated with immunosuppressive effects at standard doses, rendering issues related to dosing and timing of application critical. 
The effect of GM-CSF may be further enhanced by coadministration of IL-2. Adjuvant activity has also been attributed to IL-2, which has been widely used in clinical trials and usually is administered systemically. However, in murine tumor models GM-CSF and IL-2 were shown to act synergistically when applied intradermal in emulsion along with a peptide, leading to improved and long-lasting peptide-specific CTL responses (66).

However, care should be taken using IL-2 as it may negatively impact on anti-cancer responses (e.g., promoting the accumulation and/or activation of Tregs). Recently, attention has focused on another cytokine belonging to the common gamma chain family: IL-21. IL-21 can exert potent anti-tumor effects due to its ability to induce and expand $\mathrm{CD}^{+}$CTLs and NK cells. Importantly, IL-21 suppresses FOXP3 expression and the expansion of regulatory T cells (Tregs). Recently, it has been shown that tumorinfiltrating lymphocytes expanded ex vivo with APCs engineered to secrete IL-21 performed better than those expanded in the presence of IL-2 (71). Moreover, the $\mathrm{CD}^{+} \mathrm{T}$ cells expanded in the presence of IL-21 exhibited a less differentiated, "young" phenotype. To date, there are no studies describing inclusion of IL-21 in therapeutic vaccines. Yet, promising results have been obtained in vitro: mature DCs transfected with IL-21 were superior in priming naïve $\mathrm{CD} 8^{+} \mathrm{T}$ cells than non-transfected DCs (72).

\section{MICROMILIEU RENDERING T CELLS DYSFUNCTIONAL}

Both, chronic antigen expression and suboptimal priming in the tumor-environment renders TA-specific T cells dysfunctional. Chronic exposure to TA leads to exhausted T cells while suboptimal priming due to poor antigen presentation at tumor sites drives T cells into anergy $(73,74)$. These different aspects of T cell function can be discerned by addressing expression of specific sets of inhibitory receptors on TA-specific T cells. TA-specific CTLs present in peripheral blood lymphocytes (PBL) or at tumor sites have been shown to up-regulate $\mathrm{PD}-1$ expression, which regulates their expansion (75-77). Next to PD-1, also the inhibitory receptors Tim-3 and LAG-3 can be upregulated on tumor-infiltrating T cells and serve as markers for exhausted T cells. By contrast, anergic T cells are characterized by BTLA expression (78). Notably, BTLA has been detected on spontaneous Mart-1- and NY-ESO1-specific $\mathrm{CD}^{+} \mathrm{T}$ cells in advanced melanoma patients $(79,80)$.

Expression levels of PD-1 on exhausted T cells correlate with inhibition of different aspects of CTL function (81). As blocking Abs display most affinity for PD- $1^{\text {high }}$ expressing cells, functions inhibited due to low and/or intermediate PD-1 levels will not be regained (i.e., IL-2, TNF- $\alpha$ production and proliferation and cytotoxic activity, and IFN- $\gamma$ production, respectively). The observation that PD-1 block does not alleviate the function of TA-specific CTLs on a per-cell basis argues in favor of combining this strategy with blocking other immune check-point inhibitors. Indeed, studies performed in patients and in mice with advanced melanoma showed that blockade of both PD-1 and Tim-3 acts synergistically to enhance TA-specific $\mathrm{CD}^{+}{ }^{+} \mathrm{T}$ cell numbers and functions, resulting in decreased tumor growth (82-84). Likewise, combining Lag-3 blockade with PD-1 blockade may enhance activity of PD-1 blockade.

It has been shown that TA-specific $\mathrm{CD}^{+} \mathrm{T}$ cells exhibited variable levels of dysfunction, which correlated with a specific expression pattern of markers (80). BTLA blockade has been shown to act in concert with PD-1 and Tim-3 blockades to further enhance NY-ESO-1-specific CD8 ${ }^{+} \mathrm{T}$ cell expansion and function (80). The specific combination of inhibitory and anergy-related molecules might indicate a hierarchical loss of $\mathrm{T}$ cell function in patients with advanced melanoma in context of chronic antigen stimulation. Moreover, BTLA expression is inversely correlated with CD8 $\mathrm{T}$ cell maturation and thus anergic $\mathrm{BTLA}^{+} \mathrm{T}$ cells are likely to represent young TA-specific CTLs. Recently, a positive association of CD8 ${ }^{+}$T cells expressing BTLA with clinical response to adoptive $\mathrm{T}$ cell therapy in late-stage melanoma patients has been suggested by Haymaker (85).

Alternatively, these approaches may be even more active when combined with other agents that activate or inhibit key molecular regulators of $\mathrm{T}$ cell function, such as, for example, the tryptophan converting enzyme indoleamine-2,3-dioxygenase (IDO) and the membrane-bound CD39 and CD73 that breakdown arginase. IDO is highly expressed in both tumor cells and immune cells in the tumor-environment and implicated in inhibiting anti-tumor immunity by promoting the induction of anergic and/or regulatory $\mathrm{T}$ cells (86-89). Importantly, using pre-clinical animal melanoma models it was recently shown that IDO is responsible for mediating resistance to anti-CTLA-4 and anti-PD-1 therapy (90). Two IDO inhibitors have entered clinical trials: the tryptophan analog 1-methyl-tryptophan (1-MT) and the enzymatic inhibitor of IDO termed INCB024360. Both IDO inhibitors have been effective in pre-clinical models, attenuating tumor growth in wild type but not immuno-deficient mice $(91,92)$. INCB024360 has now entered Phase II trials, where it will be tested as a monotherapy in ovarian cancer and as a combination therapy with ipilimumab (anti-CTLA-4) for metastatic melanoma (Figure 2).

\section{IMPLICATIONS FOR THERAPY DESIGN - FUTURE DIRECTIONS}

Our understanding in the mechanism of cross-presentation is crucial in the design of vaccination strategies aimed to induce protective immunity in the field of infectious diseases and cancer, which depends on the induction of both effector $\mathrm{CD} 4^{+}$and $\mathrm{CD}^{+} \mathrm{T}$ cells. Enhanced immune protection was obtained by long synthetic peptides compared to short peptides, which required cross-presentation of DC, resulting in long-lasting T cell stimulation that leads to the eradication of tumors $(93,94)$. Studies on improving cross-presentation-based vaccinations have emerged as a promising tool for immune intervention, based on many human in vitro studies and murine in vivo work. Strong focus on DCtargeting receptors in vivo that mediate endocytosis show potential of efficient induction of $\mathrm{CD}^{+} \mathrm{T}$ cell cross-priming, but can also lead to $\mathrm{CD}^{+} \mathrm{T}$ cell cross-tolerance. This fine tuning between the induction of immunity or tolerance is dictated by the various parameters that affect cross-presentation as mentioned under the Sections "Human DC Subsets and Antigen Cross-Presentation and Factors Determining Cross-Presentation," the vaccine formulation, DC subset, receptor-targeting, endocytosis, and maturation stimuli. Many in vivo DC-targeting studies have been performed in mice that have demonstrated effective induction of tumor $\mathrm{CD}^{+}$ effector T cell responses through targeting of CLRs, such as CD205, 


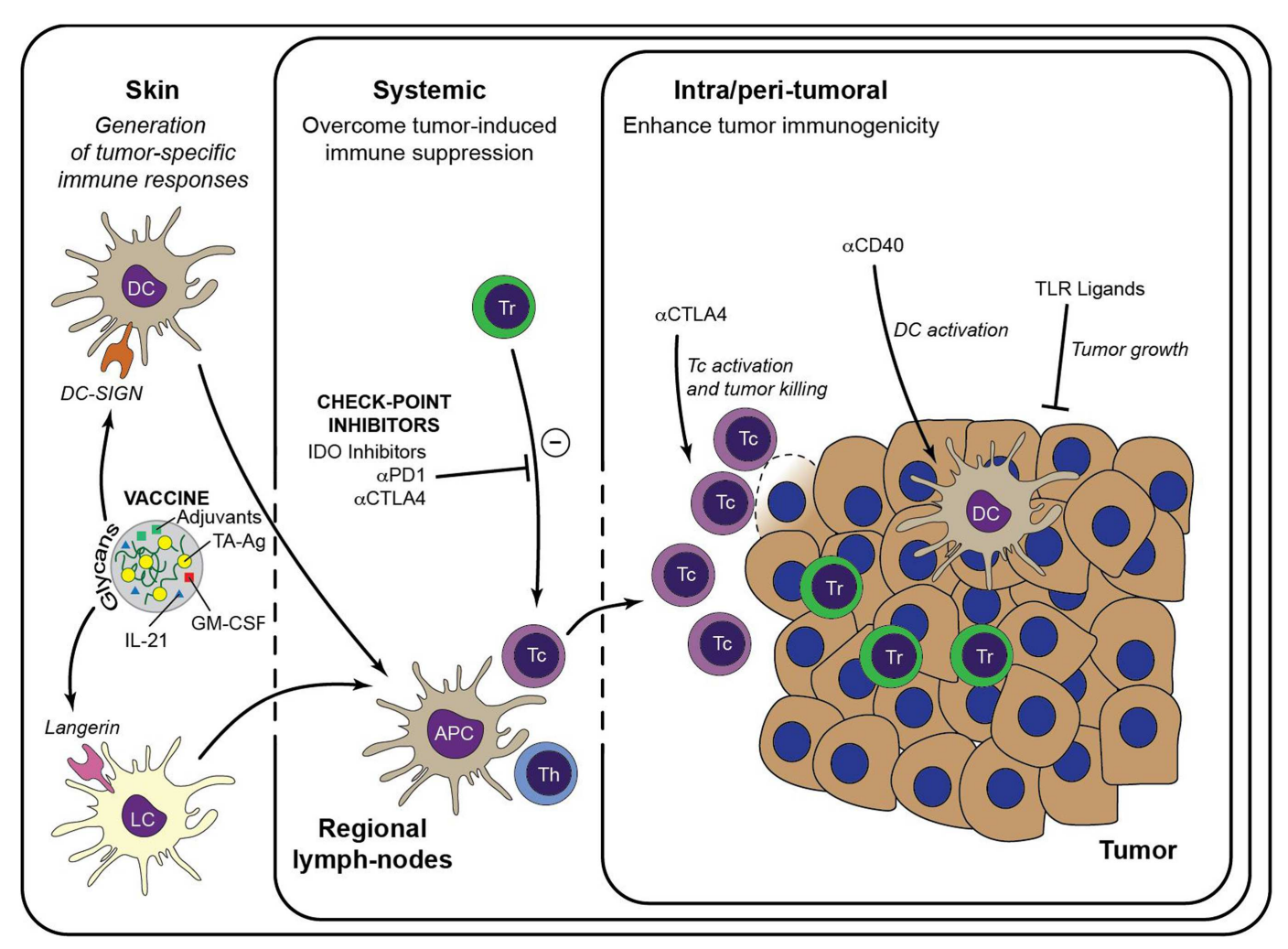

FIGURE 2 | Immunotherapeutic strategies to enhance anti-tumor immunity. Generation of a large pool of effector TA-specific T cells is induced by the intradermal injection of anti-tumor vaccines. Targeting of the vaccine to a particular skin DC subset is facilitated by modification with specific glycans that bind either to DC-SIGN or Langerin. Subsequent vaccine internalization induces presentation of TA-Ag and maturation of the DCs. Matured DCs migrate to draining lymph nodes to prime TA-specific $\mathrm{CD}^{+} \mathrm{T}$ cells and $\mathrm{CD} 4^{+} \mathrm{T}$ helper cells, leading to a large pool of cytotoxic effector $T$ cells that are capable to infiltrate the tumor lesion and lyse tumor cells. Priming of TA-specific T cells may be enhanced by inclusion of immunostimulators such as GM-CSF and IL-21 in the DC-targeting vaccine. Systemic or intra-tumoral administered check-point inhibitors, such as anti-PD-1 and anti-CTLA-4, release the break on the anti-tumor immune response by limiting the activity of suppressive Treg and alleviate the priming and/or function of TA-specific CTLs. Similarly, anti-tumor immunity may be enhanced by manipulation of the local micromilieu via administration of DC activating antibodies (e.g., anti-CD40) or of TLR ligands that act directly on the tumor cells. It is anticipated that these strategies may enhance the efficacy of DC-targeted vaccination. Tc, cytotoxic CD8 ${ }^{+} \mathrm{T}$ cell; Th, T helper cell.
MR, CD207 (Langerin), CD209 (DC-SIGN), CLEC9A or other cell-surface receptors like integrins, HSP receptors, and glycolipids. In contrast, only a few of these DC-targeting vaccines have been tested in human clinical trails. Easy translation from mouse models to humans is complicated by the different expression levels of DC-targeting receptors and DC restriction and usage of TLRs between mouse and human. Moreover, still little is known on the cross-presenting capacity of human DC in situ. This has hampered the development of novel-targeted vaccination strategies for clinical applications, and is a complicated task to fulfill in the coming years. Highly effective DC-targeting therapies should overcome the mechanism of immune escape dictated by the tumor microenvironment. Therefore, combined regimens consisting of strategies to improve DC-induced T cell responses, increasing the frequency of anti-tumor $\mathrm{T}$ cells, reversing $\mathrm{T}$ cell exhaustion that stimulate trafficking to the tumor site, along with a blockade of immuneinhibitory pathways, all may be necessary to achieve clinical benefit for cancer patients.

\section{REFERENCES}

1. Joffre OP, Segura E, Savina A, Amigorena S. Cross-presentation by dendritic cells. Nat Rev Immunol (2012) 12:557-69. doi:10.1038/nri3254

2. Delamarre L, Mellman I. Harnessing dendritic cells for immunotherapy. Semin Immunol (2011) 23:2-11. doi:10.1016/j.smim.2011.02.001

3. Barrio MM, Abes R, Colombo M, Pizzurro G, Boix C, Roberti MP, et al. Human macrophages and dendritic cells can equally present MART-1 antigen to CD8 $(+)$ T cells after phagocytosis of gamma-irradiated melanoma cells. PLoS One (2012) 7:e40311. doi:10.1371/journal.pone.0040311

4. Leiriao P, del FC, Ardavin C. Monocytes as effector cells: activated Ly-6C(high) mouse monocytes migrate to the lymph nodes through the lymph and cross-present antigens to CD8+ T cells. Eur J Immunol (2012) 42:2042-51. doi:10.1002/eji.201142166

5. Milo I, Sapoznikov A, Kalchenko V, Tal O, Krauthgamer R, van Rooijen N, et al. Dynamic imaging reveals promiscuous crosspresentation of blood-borne antigens to naive CD8+ T cells in the bone marrow. Blood (2013) 122:193-208. doi:10.1182/blood-2012-01-401265

6. Nierkens S, Tel J, Janssen E, Adema GJ. Antigen cross-presentation by dendritic cell subsets: one general or all sergeants? Trends Immunol (2013) 34:361-70. doi:10.1016/j.it.2013.02.007

7. Rocha N, Neefjes J. MHC class II molecules on the move for successful antigen presentation. EMBO J (2008) 27:1-5. doi:10.1038/sj.emboj.7601945 
8. Vyas JM, Van der Veen AG, Ploegh HL. The known unknowns of antigen processing and presentation. Nat Rev Immunol (2008) 8:607-18. doi:10.1038/nri2368

9. Blum JS, Wearsch PA, Cresswell P. Pathways of antigen processing. Annu Rev Immunol (2013) 31:443-73. doi:10.1146/annurev-immunol-032712-095910

10. Yewdell JW. DRiPs solidify: progress in understanding endogenous MHC class I antigen processing. Trends Immunol (2011) 32:548-58. doi:10.1016/j.it.2011. 08.001

11. Colbert JD, Farfan-Arribas DJ, Rock KL. Substrate-induced protein stabilization reveals a predominant contribution from mature proteins to peptides presented on MHC class I. J Immunol (2013) 191:5410-9. doi:10.4049/jimmunol.1300078

12. Kim SH, Visser A, Cruijsen C, van der Velden AW, Boes M. Recruitment of Rab27a to phagosomes controls microbial antigen cross-presentation by dendritic cells. Infect Immun (2008) 76:5373-80. doi:10.1128/IAI.01044-08

13. Leibundgut-Landmann S, Osorio F, Brown GD, Reis e Sousa C. Stimulation of dendritic cells via the dectin-1/Syk pathway allows priming of cytotoxic T-cell responses. Blood (2008) 112:4971-80. doi:10.1182/blood-2008-05-158469

14. Sancho D, Joffre OP, Keller AM, Rogers NC, Martínez D, Hernanz-Falcón P, et al. Identification of a dendritic cell receptor that couples sensing of necrosis to immunity. Nature (2009) 458:899-903. doi:10.1038/nature07750

15. Peaper DR, Cresswell P. Regulation of MHC class I assembly and peptide binding. Annu Rev Cell Dev Biol (2008) 24:343-68. doi:10.1146/annurev.cellbio.24. 110707.175347

16. Kovacsovics-Bankowski M, Rock KL. A phagosome-to-cytosol pathway for exogenous antigens presented on MHC class I molecules. Science (1995) 267:243-6. doi:10.1126/science.7809629

17. Maupin-Furlow J. Proteasomes and protein conjugation across domains of life. Nat Rev Microbiol (2012) 10:100-11. doi:10.1038/nrmicro2696

18. Macagno A, Gilliet M, Sallusto F, Lanzavecchia A, Nestle FO, Groettrup M. Dendritic cells up-regulate immunoproteasomes and the proteasome regulator PA28 during maturation. Eur J Immunol (1999) 29:4037-42. doi:10.1002/(SICI)15214141(199912)29:12<4037::AID-IMMU4037>3.0.CO;2-T

19. Basler M, Kirk CJ, Groettrup M. The immunoproteasome in antigen processing and other immunological functions. Curr Opin Immunol (2013) 25:74-80. doi:10.1016/j.coi.2012.11.004

20. Saveanu L, Carroll O, Lindo V, Del Val M, Lopez D, Lepelletier Y, et al. Concerted peptide trimming by human ERAP1 and ERAP2 aminopeptidase complexes in the endoplasmic reticulum. Nat Immunol (2005) 6:689-97. doi: $10.1038 /$ ni1208

21. Merzougui N, Kratzer R, Saveanu L, van Endert P. A proteasome-dependent, TAP-independent pathway for cross-presentation of phagocytosed antigen. EMBO Rep (2011) 12:1257-64. doi:10.1038/embor.2011.203

22. Ramachandra L, Simmons D, Harding CV. MHC molecules and microbial antigen processing in phagosomes. Curr Opin Immunol (2009) 21:98-104. doi:10.1016/j.coi.2009.01.001

23. Segura E, Villadangos JA. A modular and combinatorial view of the antigen cross-presentation pathway in dendritic cells. Traffic (2011) 12:1677-85. doi:10.1111/j.1600-0854.2011.01254.x

24. Burgdorf S, Scholz C, Kautz A, Tampe R, Kurts C. Spatial and mechanistic separation of cross-presentation and endogenous antigen presentation. Nat Immunol (2008) 9:558-66. doi:10.1038/ni.1601

25. Lampen MH, Verweij MC, Querido B, van der Burg SH, Wiertz EJ, van Hall T. CD8+ T cell responses against TAP-inhibited cells are readily detected in the human population. J Immunol (2010) 185:6508-17. doi:10.4049/jimmunol. 1001774

26. Guermonprez P, Saveanu L, Kleijmeer M, Davoust J, Van Endert P, Amigorena S. ER-phagosome fusion defines an MHC class I cross-presentation compartment in dendritic cells. Nature (2003) 425:397-402. doi:10.1038/nature01911

27. Burgdorf S, Kautz A, Bohnert V, Knolle PA, Kurts C. Distinct pathways of antigen uptake and intracellular routing in CD4 and CD8 T cell activation. Science (2007) 316:612-6. doi:10.1126/science.1137971

28. Houde M, Bertholet S, Gagnon E, Brunet S, Goyette G, Laplante A, et al. Phagosomes are competent organelles for antigen cross-presentation. Nature (2003) 425:402-6. doi:10.1038/nature01912

29. Cebrian I, Visentin G, Blanchard N, Jouve M, Bobard A, Moita C, et al. Sec22b regulates phagosomal maturation and antigen crosspresentation by dendritic cells. Cell (2011) 147:1355-68. doi:10.1016/j.cell.2011.11.021

30. Delamarre L, Pack M, Chang H, Mellman I, Trombetta ES. Differential lysosomal proteolysis in antigen-presenting cells determines antigen fate. Science (2005) 307:1630-4. doi:10.1126/science.1108003
31. Accapezzato D, Visco V, Francavilla V, Molette C, Donato T, Paroli M, et al. Chloroquine enhances human CD8+ T cell responses against soluble antigens in vivo. J Exp Med (2005) 202:817-28. doi:10.1084/jem.20051106

32. van Montfoort N, Camps MG, Khan S, Filippov DV, Weterings JJ, Griffith JM, et al. Antigen storage compartments in mature dendritic cells facilitate prolonged cytotoxic T lymphocyte cross-priming capacity. Proc Natl Acad Sci U S A (2009) 106:6730-5. doi:10.1073/pnas.0900969106

33. Cella M, Facchetti F, Lanzavecchia A, Colonna M. Plasmacytoid dendritic cells activated by influenza virus and CD40L drive a potent TH1 polarization. Nat Immunol (2000) 1:305-10. doi:10.1038/79747

34. Hoeffel G, Ripoche AC, Matheoud D, Nascimbeni M, Escriou N, Lebon P, et al. Antigen crosspresentation by human plasmacytoid dendritic cells. Immunity (2007) 27:481-92. doi:10.1016/j.immuni.2007.07.021

35. Tel J, Sittig SP, Blom RA, Cruz LJ, Schreibelt G, Figdor CG, et al. Targeting uptake receptors on human plasmacytoid dendritic cells triggers antigen crosspresentation and robust type I IFN secretion. J Immunol (2013) 191:5005-12. doi:10.4049/jimmunol.1300787

36. Poulin LF, Salio M, Griessinger E, Anjos-Afonso F, Craciun L, Chen JL, et al. Characterization of human DNGR-1+ BDCA3+ leukocytes as putative equivalents of mouse CD8alpha+ dendritic cells. J Exp Med (2010) 207:1261-71. doi:10.1084/jem.20092618

37. Crozat K, Guiton R, Contreras V, Feuillet V, Dutertre CA, Ventre E, et al. The XC chemokine receptor 1 is a conserved selective marker of mammalian cells homologous to mouse CD8alpha+ dendritic cells. J Exp Med (2010) 207:1283-92. doi:10.1084/jem.20100223

38. Bachem A, Güttler S, Hartung E, Ebstein F, Schaefer M, Tannert A, et al. Superior antigen cross-presentation and XCR1 expression define human CD11c+CD141+ cells as homologues of mouse CD8+ dendritic cells. J Exp Med (2010) 207:1273-81. doi:10.1084/jem.20100348

39. Jongbloed SL, Kassianos AJ, McDonald KJ, Clark GJ, Ju X, Angel CE, et al. Human CD141+ (BDCA-3) + dendritic cells (DCs) represent a unique myeloid DC subset that cross-presents necrotic cell antigens. J Exp Med (2010) 207:1247-60. doi:10.1084/jem.20092140

40. van de Ven R, van den Hout MF, Lindenberg JJ, Sluijter BJ, van Leeuwen PA, Lougheed SM, et al. Characterization of four conventional dendritic cell subsets in human skin-draining lymph nodes in relation to T-cell activation. Blood (2011) 118:2502-10. doi:10.1182/blood-2011-03-344838

41. Haniffa M, Shin A, Bigley V, McGovern N, Teo P, See P, et al. Human tissues contain CD141hi cross-presenting dendritic cells with functional homology to mouse CD103+ nonlymphoid dendritic cells. Immunity (2012) 37:60-73. doi:10.1016/j.immuni.2012.04.012

42. Cohn L, Chatterjee B, Esselborn F, Smed-Sörensen A, Nakamura N, Chalouni $\mathrm{C}$, et al. Antigen delivery to early endosomes eliminates the superiority of human blood BDCA3+ dendritic cells at cross presentation. J Exp Med (2013) 210:1049-63. doi:10.1084/jem.20121251

43. Nestle FO, Zheng XG, Thompson CB, Turka LA, Nickoloff BJ. Characterization of dermal dendritic cells obtained from normal human skin reveals phenotypic and functionally distinctive subsets. J Immunol (1993) 151:6535-45.

44. Klechevsky E, Morita R, Liu M, Cao Y, Coquery S, Thompson-Snipes L, et al. Functional specializations of human epidermal Langerhans cells and CD14+ dermal dendritic cells. Immunity (2008) 29:497-510. doi:10.1016/j.immuni. 2008.07.013

45. Segura E, Valladeau-Guilemond J, Donnadieu MH, Sastre-Garau X, Soumelis $\mathrm{V}$, Amigorena S. Characterization of resident and migratory dendritic cells in human lymph nodes. J Exp Med (2012) 209:653-60. doi:10.1084/jem.20111457

46. Chu CC, Ali N, Karagiannis P, Di Meglio P, Skowera A, Napolitano L, et al. Resident CD141 (BDCA3)+ dendritic cells in human skin produce IL-10 and induce regulatory $\mathrm{T}$ cells that suppress skin inflammation. J Exp Med (2012) 209:935-45. doi:10.1084/jem.20112583

47. Mittag D, Proietto AI, Loudovaris T, Mannering SI, Vremec D, Shortman K, et al. Human dendritic cell subsets from spleen and blood are similar in phenotype and function but modified by donor health status. J Immunol (2011) 186:6207-17. doi:10.4049/jimmunol.1002632

48. Klechevsky E, Flamar AL, Cao Y, Blanck JP, Liu M, O’Bar A, et al. Cross-priming CD8+ T cells by targeting antigens to human dendritic cells through DCIR. Blood (2010) 116:1685-97. doi:10.1182/blood-2010-01-264960

49. Nizzoli G, Krietsch J, Weick A, Steinfelder S, Facciotti F, Gruarin P, et al. Human $\mathrm{CD} 1 \mathrm{c}+$ dendritic cells secrete high levels of IL-12 and potently prime cytotoxic T-cell responses. Blood (2013) 122:932-42. doi:10.1182/blood-2013-04-495424 
50. Schnurr M, Chen Q, Shin A, Chen W, Toy T, Jenderek C, et al. Tumor antigen processing and presentation depend critically on dendritic cell type and the mode of antigen delivery. Blood (2005) 105:2465-72. doi:10.1182/blood-200408-3105

51. Flinsenberg TW, Compeer EB, Koning D, Klein M, Amelung FJ, van Baarle $\mathrm{D}$, et al. Fcgamma receptor antigen targeting potentiates cross-presentation by human blood and lymphoid tissue BDCA-3+ dendritic cells. Blood (2012) 120:5163-72. doi:10.1182/blood-2012-06-434498

52. van Kooyk Y. C-type lectins on dendritic cells: key modulators for the induction of immune responses. Biochem Soc Trans (2008) 36:1478-81. doi:10.1042/ BST0361478

53. Schreibelt G, Klinkenberg LJ, Cruz LJ, Tacken PJ, Tel J, Kreutz M, et al. The Ctype lectin receptor CLEC9A mediates antigen uptake and (cross-)presentation by human blood BDCA3+ myeloid dendritic cells. Blood (2012) 119:2284-92. doi:10.1182/blood-2011-08-373944

54. Chatterjee B, Smed-Sörensen A, Cohn L, Chalouni C, Vandlen R, Lee BC, et al. Internalization and endosomal degradation of receptor-bound antigens regulate the efficiency of cross presentation by human dendritic cells. Blood (2012) 120:2011-20. doi:10.1182/blood-2012-01-402370

55. Poulin LF, Reyal Y, Uronen-Hansson H, Schraml BU, Sancho D, Murphy KM, et al. DNGR-1 is a specific and universal marker of mouse and human Batf3dependent dendritic cells in lymphoid and nonlymphoid tissues. Blood (2012) 119:6052-62. doi:10.1182/blood-2012-01-406967

56. Unger WW, van Beelen AJ, Bruijns SC, Joshi M, Fehres CM, van Bloois L, et al. Glycan-modified liposomes boost CD4+ and CD8+ T-cell responses by targeting DC-SIGN on dendritic cells. J Control Release (2012) 160:88-95. doi:10.1016/j.jconrel.2012.02.007

57. García-Vallejo JJ, Ambrosini M, Overbeek A, van Riel WE, Bloem K, Unger WW, et al. Multivalent glycopeptide dendrimers for the targeted delivery of antigens to dendritic cells. Mol Immunol (2013) 53:387-97. doi:10.1016/j.molimm.2012. 09.012

58. van der Vlist $M$, de Witte L, de Vries RD, Litjens M, de Jong MA, Fluitsma D, et al. Human Langerhans cells capture measles virus through Langerin and present viral antigens to $\mathrm{CD} 4(+) \mathrm{T}$ cells but are incapable of crosspresentation. Eur J Immunol (2011) 41:2619-31. doi:10.1002/eji.201041305

59. Oosterhoff D, Heusinkveld M, Lougheed SM, Kosten I, Lindstedt M, Bruijns SC, et al. Intradermal delivery of TLR agonists in a human explant skin model: preferential activation of migratory dendritic cells by polyribosinicpolyribocytidylic acid and peptidoglycans. J Immunol (2013) 190:3338-45. doi:10.4049/jimmunol.1200598

60. Walter A, Schäfer M, Cecconi V, Matter C, Urosevic-Maiwald M, Belloni B, et al. Aldara activates TLR7-independent immune defence. Nat Commun (2013) 4:1560. doi: $10.1038 /$ ncomms 2566

61. Tillman DK Jr, Carroll MT. Topical imiquimod therapy for basal and squamous cell carcinomas: a clinical experience. Cutis (2007) 79:241-8.

62. Fransen MF, Sluijter M, Morreau H, Arens R, Melief CJ. Local activation of CD8 $\mathrm{T}$ cells and systemic tumor eradication without toxicity via slow release and local delivery of agonistic CD40 antibody. Clin Cancer Res (2011) 17:2270-80. doi:10.1158/1078-0432.CCR-10-2888

63. Schmidt J, Welsch T, Jäger D, Mühlradt PF, Büchler MW, Märten A. Intratumoural injection of the toll-like receptor-2/6 agonist 'macrophage-activating lipopeptide-2' in patients with pancreatic carcinoma: a phase I/II trial. Br J Cancer (2007) 97:598-604. doi:10.1038/sj.bjc.6603903

64. Salaun B, Romero P, Lebecque S. Toll-like receptors' two-edged sword: when immunity meets apoptosis. Eur J Immunol (2007) 37:3311-8. doi:10.1002/eji. 200737744

65. Salaun B, Lebecque S, Matikainen S, Rimoldi D, Romero P. Toll-like receptor 3 expressed by melanoma cells as a target for therapy? Clin Cancer Res (2007) 13:4565-74. doi:10.1158/1078-0432.CCR-07-0274

66. Toubaji A, Hill S, Terabe M, Qian J, Floyd T, Simpson RM, et al. The combination of GM-CSF and IL-2 as local adjuvant shows synergy in enhancing peptide vaccines and provides long term tumor protection. Vaccine (2007) 25:5882-91. doi:10.1016/j.vaccine.2007.05.040

67. Disis ML, Bernhard H, Shiota FM, Hand SL, Gralow JR, Huseby ES, et al. Granulocyte-macrophage colony-stimulating factor: an effective adjuvant for protein and peptide-based vaccines. Blood (1996) 88:202-10.

68. Nemunaitis J. Vaccines in cancer: GVAX, a GM-CSF gene vaccine. Expert Rev Vaccines (2005) 4:259-74. doi:10.1586/14760584.4.3.259
69. Santegoets SJ, Stam AG, Lougheed SM, Gall H, Scholten PE, Reijm M, et al. T cell profiling reveals high CD4+CTLA $-4+\mathrm{T}$ cell frequency as dominant predictor for survival after prostate GVAX/ipilimumab treatment. Cancer Immunol Immunother (2013) 62:245-56. doi:10.1007/s00262-012-1330-5

70. van Elsas A, Hurwitz AA, Allison JP. Combination immunotherapy of B16 melanoma using anti-cytotoxic T lymphocyte-associated antigen 4 (CTLA-4) and granulocyte/macrophage colony-stimulating factor (GM-CSF)-producing vaccines induces rejection of subcutaneous and metastatic tumors accompanied by autoimmune depigmentation. J Exp Med (1999) 190:355-66.

71. Santegoets SJ, Turksma AW, Suhoski MM, Stam AG, Albelda SM, Hooijberg E, et al. IL-21 promotes the expansion of CD27+ CD28+ tumor infiltrating lymphocytes with high cytotoxic potential and low collateral expansion of regulatory T cells. J Transl Med (2013) 11:37. doi:10.1186/1479-5876-11-37

72. Turksma AW, Bontkes HJ, Ruizendaal JJ, van den Heuvel H, Scholten KB, Santegoets SJ, et al. Increased cytotoxic capacity of tumor antigen specific human $\mathrm{T}$ cells after in vitro stimulation with IL21 producing dendritic cells. Hum Immunol (2013) 74:506-13. doi:10.1016/j.humimm.2013.01.014

73. Kim PS, Ahmed R. Features of responding T cells in cancer and chronic infection. Curr Opin Immunol (2010) 22:223-30. doi:10.1016/j.coi.2010.02.005

74. Crespo J, Sun H, Welling TH, Tian Z, Zou W. T cell anergy, exhaustion, senescence, and stemness in the tumor microenvironment. Curr Opin Immunol (2013) 25:214-21. doi:10.1016/j.coi.2012.12.003

75. Fourcade J, Kudela P, Sun Z, Shen H, Land SR, Lenzner D, et al. PD- 1 is a regulator of NY-ESO-1-specific CD8+ T cell expansion in melanoma patients. J Immunol (2009) 182:5240-9. doi:10.4049/jimmunol.0803245

76. Ahmadzadeh M, Johnson LA, Heemskerk B, Wunderlich JR, Dudley ME, White DE, et al. Tumor antigen-specific CD8 $\mathrm{T}$ cells infiltrating the tumor express high levels of PD-1 and are functionally impaired. Blood (2009) 114:1537-44. doi:10.1182/blood-2008-12-195792

77. Matsuzaki J, Gnjatic S, Mhawech-Fauceglia P, Beck A, Miller A, Tsuji T, et al. Tumor-infiltrating NY-ESO-1-specific CD8+ T cells are negatively regulated by LAG-3 and PD-1 in human ovarian cancer. Proc Natl Acad Sci U S A (2010) 107:7875-80. doi:10.1073/pnas.1003345107

78. Hurchla MA, Sedy JR, Gavrieli M, Drake CG, Murphy TL, Murphy KM. B and T lymphocyte attenuator exhibits structural and expression polymorphisms and is highly Induced in anergic CD4+ T cells. J Immunol (2005) 174:3377-85.

79. Derré L, Rivals JP, Jandus C, Pastor S, Rimoldi D, Romero P, et al. BTLA mediates inhibition of human tumor-specific CD8+ T cells that can be partially reversed by vaccination. J Clin Invest (2010) 120:157-67. doi:10.1172/JCI40070

80. Fourcade J, Sun Z, Pagliano O, Guillaume P, Luescher IF, Sander C, et al. CD8(+) T cells specific for tumor antigens can be rendered dysfunctional by the tumor microenvironment through upregulation of the inhibitory receptors BTLA and PD-1. Cancer Res (2012) 72:887-96. doi:10.1158/0008-5472.CAN11-2637

81. Wei F, Zhong S, Ma Z, Kong H, Medvec A, Ahmed R, et al. Strength of PD-1 signaling differentially affects T-cell effector functions. Proc Natl Acad Sci U S A (2013) 110:E2480-9. doi:10.1073/pnas.1305394110

82. Fourcade J, Sun Z, Benallaoua M, Guillaume P, Luescher IF, Sander C, et al. Upregulation of Tim-3 and PD-1 expression is associated with tumor antigenspecific CD8+ T cell dysfunction in melanoma patients. J Exp Med (2010) 207:2175-86. doi:10.1084/jem.20100637

83. Sakuishi K, Apetoh L, Sullivan JM, Blazar BR, Kuchroo VK, Anderson AC. Targeting Tim-3 and PD-1 pathways to reverse T cell exhaustion and restore antitumor immunity. J Exp Med (2010) 207:2187-94. doi:10.1084/jem.20100643

84. Woo SR, Turnis ME, Goldberg MV, Bankoti J, Selby M, Nirschl CJ, et al. Immune inhibitory molecules LAG-3 and PD-1 synergistically regulate T-cell function to promote tumoral immune escape. Cancer Res (2012) 72:917-27. doi:10.1158/0008-5472.CAN-11-1620

85. Haymaker C, Wu R, Ritthipichai K, Bernatchez C, Forget M, Chen JQ, et al. Uncovering a novel function of BTLA on tumor-infiltrating CD8+ T cells. J Immuno Therapy Cancer (2013) 1(Suppl 1):O1. doi:10.1186/2051-14261-S1-O1

86. Spranger S, Spaapen RM, Zha Y, Williams J, Meng Y, Ha TT, et al. Upregulation of $\mathrm{PD}-\mathrm{L} 1, \mathrm{IDO}$, and $\mathrm{T}$ (regs) in the melanoma tumor microenvironment is driven by CD8(+) T cells. Sci Transl Med (2013) 5:200ra116. doi:10.1126/scitranslmed.3006504

87. Muller AJ, DuHadaway JB, Donover PS, Sutanto-Ward E, Prendergast GC. Inhibition of indoleamine 2,3-dioxygenase, an immunoregulatory target of the 
cancer suppression gene Bin1, potentiates cancer chemotherapy. Nat Med (2005) 11:312-9. doi:10.1038/nm1196

88. Uyttenhove C, Pilotte L, Théate I, Stroobant V, Colau D, Parmentier N, et al. Evidence for a tumoral immune resistance mechanism based on tryptophan degradation by indoleamine 2,3-dioxygenase. Nat Med (2003) 9:1269-74. doi: $10.1038 / \mathrm{nm} 934$

89. Munn DH, Mellor AL. Indoleamine 2,3-dioxygenase and tumor-induced tolerance. J Clin Invest (2007) 117:1147-54. doi:10.1172/JCI31178

90. Holmgaard RB, Zamarin D, Munn DH, Wolchok JD, Allison JP. Indoleamine 2,3dioxygenase is a critical resistance mechanism in antitumor $\mathrm{T}$ cell immunotherapy targeting CTLA-4. J Exp Med (2013) 210:1389-402. doi:10.1084/jem. 20130066

91. Liu X, Shin N, Koblish HK, Yang G, Wang Q, Wang K, et al. Selective inhibition of IDO1 effectively regulates mediators of antitumor immunity. Blood (2010) 115:3520-30. doi:10.1182/blood-2009-09-246124

92. Koblish HK, Hansbury MJ, Bowman KJ, Yang G, Neilan CL, Haley PJ, et al. Hydroxyamidine inhibitors of indoleamine-2,3-dioxygenase potently suppress systemic tryptophan catabolism and the growth of IDO-expressing tumors. Mol Cancer Ther (2010) 9:489-98. doi:10.1158/1535-7163.MCT-090628

93. Zwaveling S, Ferreira Mota SC, Nouta J, Johnson M, Lipford GB, Offringa $\mathrm{R}$, et al. Established human papillomavirus type 16-expressing tumors are effectively eradicated following vaccination with long peptides. J Immunol (2002) 169:350-8.

94. Faure F, Mantegazza A, Sadaka C, Sedlik C, Jotereau F, Amigorena S. Longlasting cross-presentation of tumor antigen in human DC. Eur J Immunol (2009) 39:380-90. doi:10.1002/eji.200838669

Conflict of Interest Statement: The authors declare that the research was conducted in the absence of any commercial or financial relationships that could be construed as a potential conflict of interest.

Received: 29 January 2014; accepted: 21 March 2014; published online: 08 April 2014. Citation: Fehres CM, Unger WWJ, Garcia-Vallejo JJ and van Kooyk Y (2014) Understanding the biology of antigen cross-presentation for the design of vaccines against cancer. Front. Immunol. 5:149. doi: 10.3389/fimmu.2014.00149

This article was submitted to Antigen Presenting Cell Biology, a section of the journal Frontiers in Immunology.

Copyright (c) 2014 Fehres, Unger, Garcia-Vallejo and van Kooyk. This is an open-access article distributed under the terms of the Creative Commons Attribution License (CC BY). The use, distribution or reproduction in other forums is permitted, provided the original author(s) or licensor are credited and that the original publication in this journal is cited, in accordance with accepted academic practice. No use, distribution or reproduction is permitted which does not comply with these terms. 